\title{
Automorphisms and Inner Automorphisms
}

\author{
Ameer Jaber and Moh'D Yasein \\ Department of Mathematics, The Hashemite University, Zarqa 13115, Jordan \\ Correspondence should be addressed to Ameer Jaber; ameerj@hu.edu.jo
}

Received 25 August 2015; Accepted 31 December 2015

Academic Editor: Shaofang Hong

Copyright (C) 2016 A. Jaber and M. Yasein. This is an open access article distributed under the Creative Commons Attribution License, which permits unrestricted use, distribution, and reproduction in any medium, provided the original work is properly cited.

Let $K$ be a field of characteristic not 2 and let $\mathscr{A}=\mathscr{A}_{0}+\mathscr{A}_{1}$ be central simple superalgebra over $K$, and let $*$ be superinvolution on $\mathscr{A}$. Our main purpose is to classify the group of automorphisms and inner automorphisms of $(\mathscr{A}, *)$ (i.e., commuting with $*)$ by using the classical theorem of Skolem-Noether. Also we study two examples of groups of automorphisms and inner automorphisms on even central simple superalgebras with superinvolutions.

\section{Introduction}

An associative super ring $R=R_{\overline{0}}+R_{\overline{1}}$ is nothing but a $(\mathbb{Z} / 2 \mathbb{Z})$ graded associative ring. A $(\mathbb{Z} / 2 \mathbb{Z})$-graded ideal $I=I_{\overline{0}}+I_{\overline{1}}$ of an associative super ring $R$ is called a superideal of $R$. An associative super ring $R$ is simple if it has no nontrivial superideals. Let $R$ be an associative super ring with $1 \in$ $R_{\overline{0}}$; then $R$ is said to be a division super ring if all nonzero homogeneous elements are invertible; that is, every $0 \neq r_{\alpha} \in$ $R_{\alpha}$ has an inverse $r_{\alpha}^{-1}$ necessarily in $R_{\alpha}$.

Let $K$ be a field of characteristic not 2; an associative $(\mathbb{Z} / 2 \mathbb{Z})$-graded $K$-algebra $\mathscr{A}=\mathscr{A}_{\overline{0}}+\mathscr{A}_{\overline{1}}$ is a finite dimensional central simple superalgebra over a field $K$, if $\widehat{Z}(\mathscr{A})=K$ is the center of $\mathscr{A}$, where

$$
\begin{aligned}
& (\widehat{Z}(\mathscr{A}))_{\alpha} \\
& \quad=\left\{a_{\alpha} \in \mathscr{A}_{\alpha} \mid a_{\alpha} b_{\beta}=(-1)^{\alpha \beta} b_{\beta} a_{\alpha} \forall b_{\beta} \in \mathscr{A}_{\beta}\right\},
\end{aligned}
$$

and the only superideals of $\mathscr{A}$ are (0) and $\mathscr{A}$ itself. We say that $\mathscr{A}_{\overline{0}} \cup \mathscr{A}_{\overline{1}}$ is the set of all homogeneous elements of $\mathscr{A}$.

Finite dimensional central simple associative superalgebras over a field $K$ are isomorphic to $\operatorname{End} V \cong M_{n}(\mathscr{D})$, where $\mathscr{D}=\mathscr{D}_{0}+\mathscr{D}_{1}$ is a finite dimensional associative division superalgebra over $K$; that is, all nonzero elements of $\mathscr{D}_{\alpha}$, $\alpha=0,1$, are invertible, and $V=V_{0}+V_{1}$ is an $n$-dimensional $\mathscr{D}$-superspace. If $\mathscr{D}_{1}=\{0\}$, the grading of $M_{n}(\mathscr{D})$ is induced by that of $V=V_{0}+V_{1}, \mathscr{A}=M_{p+q}(\mathscr{D}), p=\operatorname{dim}_{\mathscr{D}} V_{0}$, and $q=\operatorname{dim}_{\mathscr{D}} V_{1}$, so $p+q$ is a nontrivial decomposition of $n$. Meanwhile, if $\mathscr{D}_{1} \neq\{0\}$, then the grading of $M_{n}(\mathscr{D})$ is given by $\left(M_{n}(\mathscr{D})\right)_{\alpha}=M_{n}\left(\mathscr{D}_{\alpha}\right), \alpha=0,1$.

For completeness, we recall the structure theorem for central simple associative division superalgebras.

Theorem 1 (Division Superalgebra Theorem $[1,2])$. If $\mathscr{D}=$ $\mathscr{D}_{0}+\mathscr{D}_{1}$ is a finite dimensional associative division superalgebra over a field $K$, then exactly one of the following holds, where throughout $\mathscr{E}$ denotes a finite dimensional associative division algebra over $K$ :

(i) $\mathscr{D}=\mathscr{D}_{0}=\mathscr{E}$, and $\mathscr{D}_{1}=\{0\}$.

(ii) $\mathscr{D}=\mathscr{E} \otimes_{K} K[u], u^{2}=\lambda \in K^{\times}, \mathscr{D}_{0}=\mathscr{E} \otimes K 1$, and $\mathscr{D}_{1}=\mathscr{E} \otimes K u$.

(iii) $\mathscr{D}=\mathscr{E}$ or $M_{2}(\mathscr{E}) ; u \in \mathscr{D}$ such that $u^{2}=\lambda \epsilon$ $K / K^{2}\left(\lambda \in K /\left\{\alpha+\alpha^{2} \mid \alpha \in K\right\}\right.$ if $\operatorname{Char}(K)=$ 2) $\mathscr{D}_{0}=C_{\mathscr{D}}(u), \mathscr{D}_{1}=S_{\mathscr{D}}(u)$, where $C_{\mathscr{D}}(u)=\{d \in$ $\mathscr{D} \mid d u=u d\}$ and $S_{\mathscr{D}}(u)=\left\{d \in \mathscr{D} \mid d u=u^{\sigma} d\right\}$, for some quadratic Galois extension $K[u] \subseteq \mathscr{D}$ with Galois automorphism $\sigma$. Moreover, in the second case, $u=\left(\begin{array}{ll}0 & 1 \\ \lambda & 0\end{array}\right)\left(u=\left(\begin{array}{ll}0 & 1 \\ \lambda & 1\end{array}\right)\right.$ if $\left.\mathrm{Char}(K)=2\right)$ and $K[u]$ does not embed in $\mathscr{E}$.

Following [3] we say that a division superalgebra $\mathscr{D}$ is even if $Z(\mathscr{D}) \cap \mathscr{D}_{1}=\{0\}$; that is, $\mathscr{D}$ is even if its form is (i) or (iii), 
and we say that $\mathscr{D}$ is odd otherwise; that is, $\mathscr{D}$ is odd if its form is (ii). Also, if $\mathscr{A}=M_{n}(\mathscr{D})$ is a finite dimensional central simple superalgebra over a field $K$, then we say that $\mathscr{A}$ is an even $K$-superalgebra if $\mathscr{D}$ is even division superalgebra and $\mathscr{A}$ is odd $K$-superalgebra if $\mathscr{D}$ is odd division superalgebra.

In [1] Racine described all types of superinvolutions on $\mathscr{A}=M_{p+q}(\mathscr{D})$. It appears that if $*$ is a superinvolution on $\mathscr{A}$ such that $\left(\mathscr{A}_{0}, *\right)$ is simple algebra, then $p=q$ and $*$ is conjugate to the transpose involution. Otherwise, $*$ is conjugate to the orthosymplectic involution.

In [4] we proved that if $\mathscr{A}$ is a finite dimensional central simple associative superalgebra over a field $K$ of characteristic not 2 such that $\mathscr{A}$ has a superinvolution of the first kind, then $\mathscr{A}=M_{p+q}(\mathscr{D})$, where $\mathscr{D}$ is a division algebra over $K$.

In [5] we proved that $\mathscr{A}=M_{n}(\mathscr{D})$, where $\mathscr{D}_{1} \neq\{0\}$ has a pseudosuperinvolution of the first kind if and only if $\mathscr{A}$ is of order 2 in the Brauer-Wall group $\mathrm{BW}(K)$, where $K$ is a field of characteristic not 2. But if $K$ is a field of characteristic 2, and $\mathscr{A}$ is a central simple associative superalgebra over $K$, then a superinvolution (which is a pseudosuperinvolution) on $\mathscr{A}$ is just an involution on $\mathscr{A}$ respecting the grading. Moreover, if $\mathscr{A}$ is of order 2 in the Brauer-Wall group $\mathrm{BW}(K)$, then the supercenter of $\mathscr{A}$ equals the center of $\mathscr{A}$ and $\widehat{\otimes}_{K}=$ $\otimes_{K}$, which means that $\mathscr{A}$ is of order 2 in the Brauer group $\operatorname{Br}(K)$. Thus, by theorem of Albert, $\mathscr{A}$ has an involution of the first kind, but since $\mathscr{A}$ is of order 2 in the BrauerWall group $\mathrm{BW}(K), \mathscr{A}$ has superantiautomorphism of the first kind respecting the grading; therefore by [6, Chapter 8 , Theorem 8.2] $\mathscr{A}$ has an involution of the first kind respecting the grading, which means that $\mathscr{A}$ has a superinvolution (which is a pseudosuperinvolution) of the first kind if and only if $\mathscr{A}$ is of order 2 in the Brauer-Wall group BW(K).

Let $K / k$ be a separable quadratic field extension over $k$ with Galois group $\operatorname{Gal}(K / k)$, where $\operatorname{Gal}(K / k)=\{1, \sigma\}$ and $\theta^{2} \in k-k^{2}$ and $\sigma(\theta)=-\theta\left(\theta^{2} \in k /\left\{\alpha+\alpha^{2} \mid \alpha \in k\right\}\right.$, $\sigma(\theta)=\theta+1$ if $\operatorname{Char}(k)=2$ ). We recall a theorem of AlbertReihm on the existence of $K / k$-involution (involution of the second kind) which states that finite dimensional central simple algebra $\mathscr{A}$ over $K$ has a $K / k$-involution if and only if the corestriction of $\mathscr{A}$ splits over $k$. In [7] Elduque and Villa gave much better exposition and motivation for the whole theory of the existence of superinvolutions.

Throughout this work we say that if $u$ is homogeneous element in $\mathscr{A}$, then $\psi_{u}: \mathscr{A} \rightarrow \mathscr{A}$ is an inner automorphism on $\mathscr{A}$, with conjugation by $u$. Also $\operatorname{Aut}(\mathscr{A}, *)$, where $\mathscr{A}$ is a central simple superalgebra of any type, means the set of all automorphisms on $\mathscr{A}$ commuting with a given superinvolution $*$ defined on $\mathscr{A}$, and $\operatorname{in} \operatorname{Aut}(\mathscr{A}, *)$ means the set of all inner automorphisms on $\mathscr{A}$ commuting with $*$.

In this paper we examine the characterization of automorphisms and inner automorphisms on $(\mathscr{A}, *)$, where $\mathscr{A}$ is a central simple superalgebra of any type, which commute with a given superinvolution $*$ defined on $\mathscr{A}$. Then we produce two examples of even superalgebras with superinvolution to investigate in detail groups of automorphisms and inner automorphisms in these examples.

\section{Group of Automorphisms of Superalgebras}

Definition 2. Let $\mathscr{A}$ be any $K$-superalgebra; we define the map $\varphi: \mathscr{A} \rightarrow \mathscr{A}$ by

$$
a_{\alpha}^{\varphi}=(-1)^{\alpha} a_{\alpha} \quad \forall a_{\alpha} \in \mathscr{A}_{\alpha}, \alpha=\overline{0}, \overline{1} .
$$

This map, $\varphi$, is a superalgebra automorphism, called the sign automorphism, since

$$
\left(a_{\alpha} b_{\beta}\right)^{\varphi}=(-1)^{\alpha+\beta} a_{\alpha} b_{\beta}=a_{\alpha}^{\varphi} b_{\beta}^{\varphi}
$$

for all $a_{\alpha} \in \mathscr{A}_{\alpha}$ and $b_{\beta} \in \mathscr{A}_{\beta}$. The automorphism $\varphi$ has order 2 , if $\operatorname{Char}(K) \neq 2$ (unless $\mathscr{A}_{\overline{1}}=0$ ), and $\varphi=i d_{\mathscr{A}}$ if $\operatorname{Char}(K)=$ 2.

Lemma 3. Let $\mathscr{A}=\mathscr{A}_{\overline{0}}+\mathscr{A}_{\overline{0}} v$ be an odd superalgebra over a field $K$ with a superinvolution $*$, and let $\varphi$ be the sign automorphism. Then

$$
\operatorname{Aut}(\mathscr{A}, *)=\operatorname{inAut}(\mathscr{A}, *) \cup \varphi \cdot \operatorname{inAut}(\mathscr{A}, *) .
$$

Proof. If $\xi \in \operatorname{Aut}(\mathscr{A}, *)$, then by [4, Theorem 3.2] SkolemNoether theorem forces that $\xi$ or $\xi \varphi$ is inner but not both of them, so, $\xi \in \operatorname{in} \operatorname{Aut}(\mathscr{A}, *)$ or $\xi \varphi \in \operatorname{in} \operatorname{Aut}(\mathscr{A}, *)$.

Now, let $*$ be a superinvolution on an odd central simple superalgebra $\mathscr{A}=\mathscr{A}_{\overline{0}}+\mathscr{A}_{\overline{0}} v$ of any kind and let $\xi \epsilon$ $\operatorname{inAut}(\mathscr{A}, *)$. Then $\xi=\psi_{c_{\gamma}}$ for some invertible $c_{\gamma} \in \mathscr{A}_{\gamma}$. But $\left.\xi\right|_{\mathscr{A}_{\overline{0}}}$ is inner, so $\left.\xi\right|_{\mathscr{A}_{\overline{0}}}=\psi_{b_{\overline{0}}}$. Therefore, $c_{\gamma}\left(b_{\overline{0}}^{-1} a b_{\overline{0}}\right) c_{\gamma}^{-1}=a$, $\forall a \in \mathscr{A}_{\overline{0}}$, and $c_{\gamma}\left(b_{\overline{0}}^{-1} v b_{\overline{0}}\right) c_{\gamma}^{-1}=v$ which implies that $\psi_{c_{\gamma} b_{\overline{0}}^{-1}}=$ $i d_{\mathscr{A}} \Rightarrow \psi_{c_{\gamma}}=\psi_{b_{\overline{0}}}$. So, we can choose $\gamma$ to be $\overline{0}$, and hence $\psi_{c_{\overline{0}}}=\psi_{b_{\overline{0}}} \Leftrightarrow c_{\overline{0}}=\alpha b_{\overline{0}}$ for some $\alpha \in K$.

$$
\begin{gathered}
\text { Now, } a_{\alpha}^{* \psi_{b_{\overline{0}}}}=a_{\alpha}^{\psi_{b_{\overline{0}}}^{*}} \Leftrightarrow b_{\overline{0}}^{*} b_{\overline{0}}=\lambda \in K \Leftrightarrow b_{\overline{0}}^{*}=\lambda b_{\overline{0}}^{-1} \text {. Let } \\
G=\left\{b_{\overline{0}} \in \mathscr{A}_{\overline{0}}: b_{\overline{0}}^{*}=\alpha b_{\overline{0}}^{-1} \text { for some } \alpha \in K^{\times}\right\} .
\end{gathered}
$$

Let $\sim$ be a relation on $G$ defined as follows: $a_{\overline{0}} \sim b_{\overline{0}} \Leftrightarrow b_{\overline{0}} a_{\overline{0}}^{-1}=$ $\alpha \in K^{\times}$. Then one can easily show that $\sim$ is an equivalence relation on $G$, and $G / \sim=\left\{\left[b_{\overline{0}}\right] \mid b_{\overline{0}} \in G\right\}$, where $\left[b_{\overline{0}}\right]=\left\{\alpha b_{\overline{0}} \mid\right.$ $\left.\alpha \in K^{\times}\right\}$, is a group with the well-defined operation $\left[b_{\overline{0}}\right]\left[c_{\overline{0}}\right]=$ $\left[c_{\overline{0}} b_{\overline{0}}\right]$.

Theorem 4. Let $\mathscr{A}$ be an odd central simple superalgebra over a field $K$ with a superinvolution $*$; then $\operatorname{inAut}(\mathscr{A}, *) \cong G / \sim$.

Proof. Let $\delta: \operatorname{inAut}(\mathscr{A}, *) \rightarrow G / \sim$ such that $\delta\left(\psi_{b_{\overline{0}}}\right)=\left[b_{\overline{0}}\right]$; then

$$
\begin{aligned}
\delta\left(\psi_{b_{\overline{0}}} \psi_{c_{\overline{0}}}\right) & =\delta\left(\psi_{c_{\overline{0}} b_{\overline{0}}}\right)=\left[c_{\overline{0}} b_{\overline{0}}\right]=\left[b_{\overline{0}}\right]\left[c_{\overline{0}}\right] \\
& =\delta\left(\psi_{b_{\overline{0}}}\right) \delta\left(\psi_{c_{\overline{0}}}\right) .
\end{aligned}
$$

So, $\delta$ is an onto homomorphism such that $\operatorname{ker}(\delta)=\psi_{1}$. Therefore, $\operatorname{in} \operatorname{Aut}(\mathscr{A}, *) \cong G / \sim$.

Let $\mathscr{A}=M_{n}(\mathscr{D})$ be an even central simple superalgebra over $K$, where $\mathscr{D}$ is a nontrivial grading division superalgebra. Then by [1, Division Superalgebra Theorem] 
$\mathscr{D}=\mathscr{D}_{\overline{0}}+\mathscr{D}_{\overline{1}}=C_{\mathscr{D}}(u)+C_{\mathscr{D}}(u) v$, where $u v=-v u$ and $Z\left(\mathscr{A}_{\overline{0}}\right)=K[u]$ and $C_{\mathscr{D}}(u)$ is the centralizer of $u$ in $\mathscr{D}$.

Let $*$ be a superinvolution on $\mathscr{A}$, and let $\xi \in \operatorname{Aut}(\mathscr{A}, *)$. Then by the Skolem-Noether theorem there exists an invertible element $c_{\gamma} \in \mathscr{A}_{\gamma}$ such that $a^{\xi}=c_{\gamma} a c_{\gamma}^{-1}, \forall a \in \mathscr{A}$. Since $a^{\xi *}=a^{* \xi}, \forall a \in \mathscr{A}$, we have $c_{\gamma} c_{\gamma}^{*}=\lambda$ for some $\lambda \in K$. If $\left.\xi\right|_{\mathscr{A}_{\overline{0}}}=$ $\left.\psi_{c_{\gamma}}\right|_{\mathscr{A}_{\overline{0}}}$ is inner, then $\left.\xi\right|_{\mathscr{A}_{\overline{0}}}=\psi_{b_{\overline{0}}}$ for some invertible element $b_{\overline{0}} \in \mathscr{A}_{\overline{0}}$ which implies that $a^{\xi}=b_{\overline{0}} a b_{\overline{0}}^{-1}, \forall a \in \mathscr{A}_{\overline{0}}$, and therefore $\psi_{b_{\overline{0}}^{-1} c_{\gamma}}=i d_{\mathscr{A}_{\overline{0}}}$. Let $x_{\gamma}=b_{\overline{0}}^{-1} c_{\gamma}$; if $\gamma=\overline{1}$, then $x_{\gamma} a x_{\gamma}^{-1}=$ $a \forall a \in \mathscr{A}_{\overline{0}}$. But $\mathscr{A}_{\overline{1}}=\mathscr{A}_{\overline{0}} x_{\gamma}$, so $x_{\gamma}$ centralizes $\mathscr{A}_{\overline{1}}$; thus $x_{\gamma} \in Z(\mathscr{A})=K$. This is a contradiction and therefore $\gamma=\overline{0}$. Therefore, for any $\xi \in \operatorname{Aut}(\mathscr{A}, *)$ such that $\left.\xi\right|_{\mathscr{A}_{\overline{0}}}$ is inner, $\xi=\psi_{b_{\overline{0}}}$, for some invertible element $b_{\overline{0}} \in \mathscr{A}_{\overline{0}}$, and hence

$$
\operatorname{inAut}(\mathscr{A}, *)=\left\{\xi \in \operatorname{Aut}(\mathscr{A}, *): \xi=\psi_{b_{\overline{0}}}, b_{\overline{0}} \in \mathscr{A}_{\overline{0}}\right\} .
$$

Corollary 5. Let $\mathscr{A}=M_{n}(\mathscr{D})$ be an even central simple superalgebra over a field $K$, where

$$
\mathscr{D}=C_{\mathscr{D}}(u)+C_{\mathscr{D}}(u) v
$$

and $u v=-v u$ is a nontrivial grading division superalgebra, and let $*$ be a superinvolution on $\mathscr{A}$. Then

$$
\begin{aligned}
& \operatorname{Aut}(\mathscr{A}, *) \\
& = \begin{cases}\operatorname{inAut}(\mathscr{A}, *) \cup \psi_{v} \cdot \operatorname{inAut}(\mathscr{A}, *): & \text { if } \psi_{v} *=* \psi_{v} \\
\operatorname{inAut}(\mathscr{A}, *): & \text { if } \psi_{v} * \neq * \psi_{v} .\end{cases}
\end{aligned}
$$

Proof. If $\psi_{v} *=* \psi_{v}$, then for any $\xi \in \operatorname{Aut}(\mathscr{A}, *)$ such that $u^{\xi}=-u$ we get $u^{\xi \psi_{v}}=-u^{\psi_{v}}=u$ which implies that $\left.\xi \psi_{v}\right|_{\mathscr{A}_{\overline{0}}}$ is inner and so $\xi \psi_{v}=\psi_{b_{\overline{0}}}$ for some invertible $b_{\overline{0}}$ in $\mathscr{A}_{\overline{0}}$. Thus $\xi=\psi_{v} \psi_{b_{\overline{0}}}$. But if $\psi_{v} * \neq * \psi_{v}$, then it is easy to check that $\operatorname{Aut}(\mathscr{A}, *)=\operatorname{in} \operatorname{Aut}(\mathscr{A}, *)$. Therefore

$$
\begin{aligned}
& \operatorname{Aut}(\mathscr{A}, *) \\
& = \begin{cases}\operatorname{in} \operatorname{Aut}(\mathscr{A}, *) \cup \psi_{v} \cdot \operatorname{inAut}(\mathscr{A}, *): & \text { if } \psi_{v} *=* \psi_{v} \\
\operatorname{inAut}(\mathscr{A}, *): & \text { if } \psi_{v} * \neq * \psi_{v} .\end{cases}
\end{aligned}
$$

Theorem 6. Let $\mathscr{A}=M_{n}(\mathscr{D})$, where $\mathscr{D}=\mathscr{D}_{\overline{0}}+\mathscr{D}_{\overline{1}}=$ $C_{\mathscr{D}}(u)+C_{\mathscr{D}}(u) v$, and let $u v=-v u$ be a superalgebra with a superinvolution $*$. Then $\operatorname{inAut}(\mathscr{A}, *) \cong G / \sim$.

Proof. Let $\delta: \operatorname{inAut}(\mathscr{A}, *) \rightarrow G / \sim \operatorname{such}$ that $\delta\left(\psi_{b_{\overline{0}}}\right)=\left[b_{\overline{0}}\right]$. Then

$$
\begin{aligned}
\delta\left(\psi_{b_{\overline{0}}} \psi_{c_{\overline{0}}}\right) & =\delta\left(\psi_{c_{\overline{0}} b_{\overline{0}}}\right)=\left[c_{\overline{0}} b_{\overline{0}}\right]=\left[b_{\overline{0}}\right]\left[c_{\overline{0}}\right] \\
& =\delta\left(\psi_{b_{\overline{0}}}\right) \delta\left(\psi_{c_{\overline{0}}}\right) .
\end{aligned}
$$

So, $\delta$ is an onto homomorphism, where $\operatorname{ker} \delta=i d_{\mathscr{A}}=\psi_{\overline{1}}$. Therefore, $\operatorname{in} \operatorname{Aut}(\mathscr{A}, *) \cong G / \sim$.

Next we introduce this example to show that $u u^{*}$ may equal $\lambda$ with $\lambda \neq \alpha \alpha^{*}$ for any $\alpha \in K$.
Example 7. Let $k=\mathbb{Q}(e)$, let $K=k(\sqrt{2})$, and let $u=\sqrt{e} i$, $v=\sqrt[4]{2} j$, where $i^{2}=-1, j^{2}=-1$, and $i j=-j i$. Then $\mathscr{A}=$ $(K+K u) \oplus(K v+K u v)$ is a quaternion division superalgebra over field $K$. Let $*$ be a superinvolution on $\mathscr{A}$ defined by

$$
\begin{aligned}
& (a+b \sqrt{2})^{*}=a-b \sqrt{2}, \forall a, b \in k ; u^{*}=u ; v^{*}=v \text {; then } \\
& (u v)^{*}=v u=-u v .
\end{aligned}
$$

Now, $\psi_{u} \in \operatorname{Aut}(\mathscr{A}, *)$, since $u u^{*}=-e \in K$. But $u u^{*} \neq \alpha \alpha^{*}$, where $\alpha \in K$, because if

$$
-e=(a+b \sqrt{2})(a+b \sqrt{2})^{*}
$$

where $a, b \in k$, then

$$
\begin{aligned}
-e & =(a+b \sqrt{2})(a-b \sqrt{2})=a^{2}-2 b^{2} \\
& =\left(\frac{p_{1}(e)}{q_{1}(e)}\right)^{2}-2\left(\frac{p_{2}(e)}{q_{2}(e)}\right)^{2}
\end{aligned}
$$

where $p_{i}(e), q_{i}(e) \in \mathbb{Q}[e]$ and $q_{i}(e) \neq 0$. Hence,

$$
\begin{aligned}
-e\left(q_{1}(e)\right)^{2}\left(q_{2}(e)\right)^{2}= & \left(p_{1}(e) q_{2}(e)\right)^{2} \\
& -2\left(p_{2}(e) q_{1}(e)\right)^{2} .
\end{aligned}
$$

Since $\sqrt{2} \notin \mathbb{Q}$, the highest power of $e$ in the right-hand side is even, but the highest power of $e$ in the left-hand side is odd, a contradiction.

Now we try to classify the group of automorphisms of central simple superalgebras $\mathscr{A}=M_{2 p}\left(\mathscr{D}_{\overline{0}}\right)$, and $\mathscr{A}=$ $M_{p+q}\left(\mathscr{D}_{\overline{0}}\right)$, where $\mathscr{D}_{\overline{0}}$ is a division algebra over $K$ and $(p \neq q)$.

First let $\mathscr{A}=M_{2 p}\left(\mathscr{D}_{\overline{0}}\right)$, where $\mathscr{D}_{\overline{0}}$ is a division algebra over $K$, and let $*$ be any superinvolution on $\mathscr{A}$. If $\xi \epsilon$ $\operatorname{Aut}(\mathscr{A}, *)$, then, by [1, Isomorhpism Theorem], $\xi=\psi_{M_{\alpha}}$ for some invertible element $M_{\alpha} \in \mathscr{A}_{\alpha}$ and

$$
M_{\alpha} P_{\gamma}^{*} M_{\alpha}^{-1}=\left(M_{\alpha} P_{\gamma} M_{\alpha}^{-1}\right)^{*}, \quad \forall P_{\gamma} \in \mathscr{A}_{\gamma}
$$

thus,

$$
\begin{aligned}
M_{\alpha} P_{\gamma}^{*} M_{\alpha}^{-1} & =(-1)^{\alpha(\alpha+\gamma)}(-1)^{\alpha \gamma}\left(M_{\alpha}^{-1}\right)^{*} P_{\gamma}^{*} M_{\alpha}^{*} \\
& =(-1)^{\alpha}\left(M_{\alpha}^{-1}\right)^{*} P_{\gamma}^{*} M_{\alpha}^{*}=\left(M_{\alpha}^{*}\right)^{-1} P_{\gamma}^{*} M_{\alpha}^{*},
\end{aligned}
$$

which implies that $M_{\alpha}^{*} M_{\alpha} P_{\gamma}^{*} M_{\alpha}^{-1}\left(M_{\alpha}^{*}\right)^{-1}=P_{\gamma}^{*}, \forall P_{\gamma}$, and therefore $M_{\alpha}^{*} M_{\alpha}=\lambda I_{2 p}$, where $\lambda \in K^{\times}$. If $*$ is a superinvolution of the first kind, then

$$
\begin{aligned}
\left(M_{\alpha}^{*} M_{\alpha}\right)^{*} & =(-1)^{\alpha^{2}} M_{\alpha}^{*} M_{\alpha}=\left(\lambda I_{2 p}\right)^{*}=\lambda I_{2 p} \\
& =M_{\alpha}^{*} M_{\alpha} .
\end{aligned}
$$

Hence $\alpha^{2}=\overline{0}$, which implies that $\alpha=\overline{0}$ and therefore

$$
\operatorname{Aut}(\mathscr{A}, *)=\left\{\psi_{M} \mid\right.
$$

$M$ is an invertible element in $\mathscr{A}_{\overline{0}}$, with $M M^{*}$

$$
\left.=\lambda I_{2 p}\right\} \text {. }
$$


Moreover, if $*$ is a superinvolution of the second kind on $\mathscr{A}$, then

$$
\operatorname{Aut}(\mathscr{A}, *)=\left\{\psi_{M} \mid\right.
$$

$M$ is an invertible element in $\mathscr{A}_{\overline{0}}$, with $M M^{*}$

$$
\left.=\lambda I_{2 p}\right\} \cup\left\{\psi_{M} \mid\right.
$$

$M$ is an invertible element in $\mathscr{A}_{\overline{1}}$, with $M M^{*}$

$$
\left.=\gamma I_{2 p}, \gamma^{*}=-\gamma\right\} \text {. }
$$

Theorem 8. Let $\mathscr{A}=M_{2 p}\left(\mathscr{D}_{\overline{0}}\right)$, where $\mathscr{D}_{\overline{0}}$ is a division algebra over $K$, and let $*$ be any superinvolution on $\mathscr{A}$. If $*$ is a superinvolution of the first kind, then $\operatorname{Aut}(\mathscr{A}, *) \cong G / \sim$, where $G / \sim$ is the group defined in Theorem 4 . If $*$ is a superinvolution of the second kind, then $\operatorname{Aut}(\mathscr{A}, *) \cong G / \sim$, where $G=\left\{b_{\overline{0}} \in\right.$ $\left.\mathscr{A}_{\overline{0}} \mid b_{\overline{0}} b_{\overline{0}}^{*}=\alpha \in K^{\times}\right\} \cup\left\{b_{\overline{1}} \in \mathscr{A}_{\overline{1}} \mid b_{\overline{1}} b_{\overline{1}}^{*}=\lambda \epsilon\right.$ $K^{\times}$, where $\left.\lambda^{*}=-\lambda\right\}$ and the equivalence relation $\sim$ on $G$ is defined by $a_{\alpha} \sim b_{\alpha} \Leftrightarrow b_{\alpha}=\gamma a_{\alpha}$ for some $\gamma \in K^{\times}$.

Proof. If $*$ is a superinvolution of the first kind, then

$$
\delta: \operatorname{Aut}(\mathscr{A}, *) \longrightarrow \stackrel{G}{\sim}, \quad\left(\psi_{a_{\overline{0}}} \longmapsto\left[a_{\overline{0}}\right]\right)
$$

is a group isomorphism.

Similarly, if $*$ is a superinvolution of the second kind, then it is easy to check that $G / \sim$ is a group under the well-defined operation $\left[a_{\alpha}\right]\left[b_{\beta}\right]=\left[b_{\beta} a_{\alpha}\right]$, and so

$$
\delta: \operatorname{Aut}(\mathscr{A}, *) \longrightarrow \frac{G}{\sim}, \quad\left(\psi_{a_{\alpha}} \longmapsto\left[a_{\alpha}\right]\right)
$$

is a group isomorphism. Thus in all cases we $\operatorname{get} \operatorname{Aut}(\mathscr{A}, *) \cong$ G/ .

Second let $\mathscr{A}=M_{p+q}\left(\mathscr{D}_{\overline{0}}\right)$, where $\mathscr{D}_{\overline{0}}$ is a division algebra over $K$ and $(p \neq q)$. Let $*$ be any superinvolution on $\mathscr{A}$ of any kind. If $\xi \in \operatorname{Aut}(\mathscr{A}, *)$, then by [1, Isomorhpism Theorem] $\xi=\psi_{M_{\alpha}}$, for some invertible element $M_{\alpha}$ in $\mathscr{A}_{\alpha}$, since $p \neq q$, the homogeneous invertible elements in $\mathscr{A}$ are the invertible elements in $\mathscr{A}_{\overline{0}}$. Thus $\alpha=\overline{0}$, and, therefore, if $\xi \in \operatorname{Aut}(\mathscr{A}, *)$, then $\xi=\psi_{M}$, where $M$ is an invertible element in $\mathscr{A}_{\overline{0}}$. Now, if $P_{\gamma} \in \mathscr{A}_{\gamma}$, then

$$
\begin{aligned}
M P_{\gamma}^{*} M^{-1} & =\left(M P_{\gamma} M^{-1}\right)^{*}=\left(M^{-1}\right)^{*} P_{\gamma}^{*} M^{*} \\
& =\left(M^{*}\right)^{-1} P_{\gamma}^{*} M^{*} .
\end{aligned}
$$

Therefore, $M^{*} M P_{\gamma}^{*} M^{-1}\left(M^{*}\right)^{-1}=P_{\gamma}^{*}, \forall P_{\gamma} \in \mathscr{A}_{\gamma}$, and hence $M^{*} M=\lambda I_{p+q}$, where $\lambda \in K^{\times}$. Thus it is easy to check that $\operatorname{Aut}(\mathscr{A}, *)=\left\{\psi_{M} \mid M\right.$ is an invertible element in $\mathscr{A}_{\overline{0}}$, where $\left.M M^{*}=\lambda I_{p+q}\right\} \cong G / \sim$, where $G=\left\{b_{\overline{0}} \in \mathscr{A}_{\overline{0}} \mid b_{\overline{0}} b_{\overline{0}}^{*}=\right.$ $\left.\alpha \in K^{\times}\right\}$.

\section{Examples of Group of Automorphisms of Even Superalgebras}

In this section we classify in detail the groups of automorphisms of superalgebras $\mathscr{A}=M_{2 p}(\mathscr{D})$, and $\mathscr{A}=M_{p+q}(\mathscr{D})$, where $\mathscr{D}=\mathbb{W}$ or $\mathbb{R}$, where $\mathbb{U}$ is the Hamiltonian quaternion algebra and $\mathbb{R}$ is the field of real numbers.

Let $\mathscr{A}=M_{2 p}(\mathscr{D})$, where $\mathscr{D}=\mathbb{H}$ or $\mathbb{R}$, and let $*$ be a superinvolution of the first kind on $\mathscr{A}$ such that $\left(\mathscr{A}_{\overline{0}},\left.*\right|_{\mathscr{A}_{\overline{0}}}\right)$ is simple; then by [1, Proposition 13] $*$ is defined by

$$
\left(\begin{array}{ll}
a & b \\
c & d
\end{array}\right) \longmapsto\left(\begin{array}{cc}
\tilde{d} & -\tilde{b} \\
\tilde{c} & \tilde{a}
\end{array}\right),
$$

where $\sim$ is the involution on $M_{p}(\mathscr{D})$ induced by $*$. Then $H=\operatorname{Aut}(\mathscr{A}, *)=\left\{\psi_{M} \mid M\right.$ is an invertible element in $\mathscr{A}_{\overline{0}}$, where $M M^{*}=\alpha I_{2 p}$, and $\left.\alpha \in \mathbb{R}\right\}$. $I_{2 p}$.

If $\alpha>0$, then by replacing $M$ by $(1 / \sqrt{\alpha}) M$ we get $M M^{*}=$

If $\alpha<0$, then by replacing $M$ by $(1 / \sqrt{-\alpha}) M$ we get $M M^{*}=-I_{2 p}$.

So $H=\left\{\psi_{M} \mid M M^{*}=I_{2 p}\right.$ or $\left.-I_{2 p}\right\}$.

Theorem 9. In the situation described above, let

$$
G=\operatorname{Aut}\left(\mathscr{A}_{\overline{0}},\left.*\right|_{\mathscr{A}_{\overline{0}}}\right)
$$

Then $H / \mathbb{R}^{\times} \cong G_{0}$, where $G_{0}$ is the connected component of the identity in $G$.

Proof. By [8, page 593], $G=G_{0} \cup \psi_{N} G_{0}$, where $N=\left(\begin{array}{cc}0 & I_{p} \\ I_{p} & 0\end{array}\right)$ and $G_{0}$ is the connected component of the identity. Let

$$
\begin{gathered}
\pi: H \longrightarrow G_{0} \\
\left(\left.\psi_{M} \longmapsto \psi_{M}\right|_{\mathscr{A}_{\overline{0}}}\right) .
\end{gathered}
$$

Then $\pi$ is onto group homomorphism because if $g_{0} \in G_{0}$, then $g_{0}=\left.\psi_{M}\right|_{\mathscr{A}_{\overline{0}}}$ for some invertible element $M \in \mathscr{A}_{\overline{0}}$ since $G_{0}$ is the connected component of the identity. Then it is easy to check that $M M^{*}=\alpha I_{2 p}(\alpha \in \mathbb{R})$ since $\psi_{M} *=* \psi_{M}$. $I_{2 p}$.

If $\alpha>0$, then by replacing $M$ by $(1 / \sqrt{\alpha}) M$ we get $M M^{*}=$

If $\alpha<0$, then by replacing $M$ by $(1 / \sqrt{-\alpha}) M$ we get $M M^{*}=-I_{2 p}$. Therefore, $\psi_{M} \in H$ and $\pi\left(\psi_{M}\right)=g_{0}$. Also it is easy to check that $\operatorname{ker} \pi=\left\{\psi_{M} \mid M=\right.$ $\left(\begin{array}{cc}\lambda I_{p} & 0 \\ 0 & \gamma I_{p}\end{array}\right)$, where $\left.\lambda, \gamma \in \mathbb{R}\right\}$. But

$$
\begin{gathered}
\left(\begin{array}{cc}
\lambda I_{p} & 0 \\
0 & \gamma I_{p}
\end{array}\right)\left(\begin{array}{cc}
\gamma I_{p} & 0 \\
0 & \lambda I_{p}
\end{array}\right)=\left(\begin{array}{cc}
\lambda \gamma I_{p} & 0 \\
0 & \gamma \lambda I_{p}
\end{array}\right)=I_{2 p} \\
\text { or }\left(\begin{array}{cc}
\lambda I_{p} & 0 \\
0 & \gamma I_{p}
\end{array}\right)\left(\begin{array}{cc}
\gamma I_{p} & 0 \\
0 & \lambda I_{p}
\end{array}\right)=\left(\begin{array}{cc}
\lambda \gamma I_{p} & 0 \\
0 & \gamma \lambda I_{p}
\end{array}\right) \\
=-I_{2 p} .
\end{gathered}
$$

So, $\gamma=1 / \lambda$ or $-1 / \lambda$. Therefore, $\operatorname{ker} \pi=\left\{\psi_{M} \mid M=\right.$ $\left(\begin{array}{cc}\lambda I_{p} & 0 \\ 0 & (1 / \lambda) I_{p}\end{array}\right)$, or $M=\left(\begin{array}{cc}\lambda I_{p} & 0 \\ 0 & (-1 / \lambda) I_{p}\end{array}\right)$, where $\left.\lambda \in \mathbb{R}^{\times}\right\} \cong \mathbb{R}^{\times}$, since $\psi_{M}=\psi_{-M}$. Therefore $G_{0} \cong H / \operatorname{ker} \pi \cong H / \mathbb{R}^{\times}$.

In the second case, let $\mathscr{A}=M_{p+q}(\mathscr{D})$, where $\mathscr{D}=\mathbb{H}$ or $\mathbb{R}$, and let $*$ be a superinvolution of the first kind on $\mathscr{A}$ such 
that $\left(\mathscr{A}_{\overline{0}},\left.*\right|_{\mathscr{A}_{0}}\right)$ is not simple; then by [1, Proposition 14$] *$ is defined by

$$
\left(\begin{array}{ll}
x & y \\
z & w
\end{array}\right) \longmapsto\left(\begin{array}{cc}
\widetilde{x} & -N \bar{z}^{t} M^{-1} \\
M \bar{y}^{t} N^{-1} & \widetilde{w}
\end{array}\right),
$$

where $\tilde{x}=N \bar{x}^{t} N^{-1}\left(\bar{N}^{t}=-N \in M_{p}(\mathscr{D})\right)$ and $\widetilde{w}=M \bar{w}^{t} M^{-1}$ $\left(\bar{M}^{t}=M \in M_{q}(\mathscr{D})\right)$ and ${ }^{-}$is the standard involution on $\mathscr{D}$.

Since $*$ is a superinvolution of the first kind we have $H=\operatorname{Aut}(\mathscr{A}, *)=\left\{\psi_{M} \mid M\right.$ is an invertible element in $\mathscr{A}_{\overline{0}}$, where $M M^{*}=\alpha I_{p+q}$, and $\left.\alpha \in \mathbb{R}\right\}$. Similarly, $H=\left\{\psi_{M} \mid M M^{*}=I_{p+q}\right.$ or $\left.-I_{p+q}\right\}$.

We will start with the case $p \neq q$.

Theorem 10. In the situation described above, let

$$
G=\operatorname{Aut}\left(\mathscr{A}_{\overline{0}},\left.*\right|_{\mathscr{A}_{\overline{0}}}\right) .
$$

Then $H / \operatorname{ker} \pi \cong \pi(H) \leq G$, where

$$
\pi: H \longrightarrow G \quad\left(\left.\psi_{N} \longmapsto \psi_{N}\right|_{\mathscr{A}_{\overline{0}}}\right)
$$

Proof. Since $\mathscr{A}_{\overline{0}}$ is semisimple and $p \neq q$, we get $G=$ $\operatorname{Aut}\left(\mathscr{A}_{\overline{0}},\left.*\right|_{\mathscr{A}_{\overline{0}}}\right)=\left\{\psi_{M} \mid M=\left(\begin{array}{cc}A & 0 \\ 0 & B\end{array}\right)\right.$, where $A, B$ are invertible matrices\}. If $\psi_{M} \in G$, then

$$
\begin{aligned}
& \left.\psi_{M} *\right|_{\mathscr{A}_{\overline{0}}}=\left.*\right|_{\mathscr{A}_{\overline{0}}} \psi_{M} \Longleftrightarrow \\
& M M^{*}=\left(\begin{array}{cc}
\lambda I_{p} & 0 \\
0 & \gamma I_{q}
\end{array}\right), \quad(\lambda, \gamma \in \mathbb{R}) ; \text { therefore } \\
& \left.\psi_{M} *\right|_{\mathscr{A}_{\overline{0}}}=\left.*\right|_{\mathscr{A}_{\overline{0}}} \psi_{M} \Longleftrightarrow \\
& M M^{*} \in V=\left\{I_{p+q},-I_{p+q},\left(\begin{array}{cc}
I_{p} & 0 \\
0 & -I_{q}
\end{array}\right),\left(\begin{array}{cc}
-I_{p} & 0 \\
0 & I_{q}
\end{array}\right)\right\} .
\end{aligned}
$$

So $G=\left\{\psi_{M} \mid M M^{*} \in V\right\}$. Therefore,

$$
\pi: H \longrightarrow G \quad\left(\left.\psi_{N} \longmapsto \psi_{N}\right|_{\mathscr{A}_{\overline{0}}}\right)
$$

is a group homomorphism and $\operatorname{ker} \pi=\left\{\psi_{N}\left|\psi_{N}\right|_{\mathscr{A}_{\overline{0}}}=\right.$ $\left.i d_{\mathscr{A}_{\overline{0}}}\right\}=\left\{i d_{\mathscr{A}}, \psi_{M}, M=\left(\begin{array}{cc}I_{p} & 0 \\ 0 & -I_{q}\end{array}\right)\right\}$. To see this let $N=\left(\begin{array}{cc}A & 0 \\ 0 & B\end{array}\right)$ such that $\psi_{N} \in \operatorname{ker} \pi$; then $N N^{*}=I_{p+q}$ or $-I_{p+q}$ (since $\left.\psi_{N} \in H\right)$, and

$$
\left(\begin{array}{cc}
A & 0 \\
0 & B
\end{array}\right)\left(\begin{array}{cc}
X & 0 \\
0 & Y
\end{array}\right)\left(\begin{array}{cc}
A^{-1} & 0 \\
0 & B^{-1}
\end{array}\right)=\left(\begin{array}{cc}
X & 0 \\
0 & Y
\end{array}\right)
$$

if and only if $A=\lambda I_{p}$ and $B=\gamma I_{q}(\lambda, \gamma \in \mathbb{R})$; since

$$
\begin{aligned}
N N^{*} & =\left(\begin{array}{cc}
\lambda I_{p} & 0 \\
0 & \gamma I_{q}
\end{array}\right)\left(\begin{array}{cc}
\lambda I_{p} & 0 \\
0 & \gamma I_{q}
\end{array}\right)=\left(\begin{array}{cc}
\lambda^{2} I_{p} & 0 \\
0 & \gamma^{2} I_{q}
\end{array}\right) \\
& \in\left\{I_{p+q},-I_{p+q}\right\}
\end{aligned}
$$

we get $N N^{*}=I_{p+q}$. Therefore, $\lambda^{2}=\gamma^{2}=1 \Rightarrow \lambda, \gamma \in\{1,-1\}$, which implies that $\operatorname{ker} \pi=\left\{\psi_{I_{p+q}}, \psi_{-I_{p+q}}, \psi_{N}, \psi_{M}\right\}$, where $N=$ $\left(\begin{array}{cc}-I_{p} & 0 \\ 0 & I_{q}\end{array}\right)$ and $M=\left(\begin{array}{cc}I_{p} & 0 \\ 0 & -I_{q}\end{array}\right)$ and hence $\operatorname{ker} \pi=\left\{i d_{\mathscr{A}}, \psi_{M}\right\}$ since $\psi_{I_{p+q}}=\psi_{-I_{p+q}}$ and $\psi_{N}=\psi_{M}$. Therefore, $H / \operatorname{ker} \pi \cong \pi(H) \leq$ G.

Theorem 11. As in the theorem above, if $p \neq q$, then $G=$ $\left.\pi(H) \cup \psi_{M}\right|_{\mathscr{A}_{\overline{0}}} \pi(H)$ for a fixed invertible matrix $M=\left(\begin{array}{cc}A & 0 \\ 0 & B\end{array}\right)$ such that

$$
M M^{*} \in\left\{\left(\begin{array}{cc}
I_{p} & 0 \\
0 & -I_{q}
\end{array}\right),\left(\begin{array}{cc}
-I_{p} & 0 \\
0 & I_{q}
\end{array}\right)\right\} .
$$

Proof. Since $M M^{*} \neq I_{p+q},-I_{p+q}$, then $\psi_{M} \notin H$, which implies that $\left.\psi_{M}\right|_{\mathscr{A}_{\overline{0}}} \notin \pi(H)$. Let $N=\left(\begin{array}{cc}X & 0 \\ 0 & Y\end{array}\right)$ such that

$$
N N^{*} \in\left\{\left(\begin{array}{cc}
I_{p} & 0 \\
0 & -I_{q}
\end{array}\right),\left(\begin{array}{cc}
-I_{p} & 0 \\
0 & I_{q}
\end{array}\right)\right\}
$$

then

$$
\begin{aligned}
& \left(N M^{-1}\right)\left(N M^{-1}\right)^{*}=N M^{-1}\left(M^{-1}\right)^{*} N^{*} \\
& \quad=\left\{\begin{array}{l}
N\left(\begin{array}{cc}
I_{p} & 0 \\
0 & -I_{q}
\end{array}\right) N^{*}: M M^{*}=\left(\begin{array}{cc}
I_{p} & 0 \\
0 & -I_{q}
\end{array}\right) \\
N\left(\begin{array}{cc}
-I_{p} & 0 \\
0 & I_{q}
\end{array}\right) N^{*}: M M^{*}=\left(\begin{array}{cc}
-I_{p} & 0 \\
0 & I_{q}
\end{array}\right)
\end{array}\right. \\
& =\left\{\begin{array}{l}
\left(\begin{array}{cc}
X \widetilde{X} & 0 \\
0 & -Y \tilde{Y}
\end{array}\right): M M^{*}=\left(\begin{array}{cc}
I_{p} & 0 \\
0 & -I_{q}
\end{array}\right) \\
\left(\begin{array}{cc}
-X \widetilde{X} & 0 \\
0 & Y \widetilde{Y}
\end{array}\right): M M^{*}=\left(\begin{array}{cc}
-I_{p} & 0 \\
0 & I_{q}
\end{array}\right) .
\end{array}\right.
\end{aligned}
$$

Therefore, $\left(N M^{-1}\right)\left(N M^{-1}\right)^{*} \in\left\{I_{p+q},-I_{p+q}\right\}$; thus, by Theorem 10,

$$
\left.\psi_{N M^{-1}}\right|_{\mathscr{A}_{\overline{0}}}=\left.\left.\psi_{M^{-1}}\right|_{\mathscr{A}_{\overline{0}}} \psi_{N}\right|_{\mathscr{A}_{\overline{0}}} \in \pi(H) \cong \frac{H}{\operatorname{ker} \pi} .
$$

Now, $\left.\psi_{N}\right|_{\mathscr{A}_{\overline{0}}}=\left.\psi_{N M^{-1} M}\right|_{\mathscr{A}_{\overline{0}}}=\left.\left.\left.\psi_{M}\right|_{\mathscr{A}_{\overline{0}}} \psi_{M^{-1}}\right|_{\mathscr{A}_{\overline{0}}} \psi_{N}\right|_{\mathscr{A}_{\overline{0}}}=$

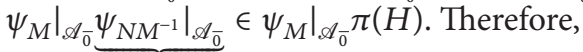

$$
G=\left.\pi(H) \cup \psi_{M}\right|_{\mathscr{A}_{\overline{0}}} \pi(H)
$$

For the case $p=q, G=\operatorname{Aut}\left(\mathscr{A}_{\overline{0}},\left.*\right|_{\mathscr{A}_{\overline{0}}}\right)=\left.G_{0} \cup \psi_{N}\right|_{\mathscr{A}_{\overline{0}}} G_{0}$, where $N=\left(\begin{array}{cc}0 & I_{p} \\ I_{p} & 0\end{array}\right)$ (it is easy to check that $\left.\left.\psi_{N}\right|_{\mathscr{A}_{\overline{0}}} *\right|_{\mathscr{A}_{\overline{0}}}=$ $\left.\left.\left.*\right|_{\mathscr{A}_{\overline{0}}} \psi_{N}\right|_{\mathscr{A}_{\overline{0}}}\right)$ and $G_{0}=\left\{\left.\psi_{M}\right|_{\mathscr{A}_{\overline{0}}} \mid M M^{*} \in V\right\}$ is the connected component of the identity in $G$. Now, let $\pi: H \rightarrow G_{0}\left(\psi_{L} \mapsto\right.$ $\left.\left.\psi_{L}\right|_{\mathscr{A}_{\overline{0}}}\right)$, where $H=\left\{\psi_{M} \mid M M^{*}=I_{p+q}\right.$ or $\left.-I_{p+q}\right\}$; then $\pi$ is a group homomorphism and $\operatorname{ker} \pi=\left\{i d_{\mathscr{A}}, \psi_{N^{\prime}}\right\}$ where $N^{\prime}=\left(\begin{array}{cc}I_{p} & 0 \\ 0 & -I_{p}\end{array}\right)$. Therefore, $H / \operatorname{ker} \pi \cong \pi(H) \leq G_{0}$. 
Theorem 12. In the situation described above, if $p=q$, then

$$
G_{0}=\left.\pi(H) \cup \psi_{M}\right|_{\mathscr{A}_{\overline{0}}} \pi(H),
$$

for a fixed invertible matrix $M=\left(\begin{array}{cc}A & 0 \\ 0 & B\end{array}\right)$, such that

$$
M M^{*} \in\left\{\left(\begin{array}{cc}
I_{p} & 0 \\
0 & -I_{q}
\end{array}\right),\left(\begin{array}{cc}
-I_{p} & 0 \\
0 & I_{q}
\end{array}\right)\right\},
$$

and $G_{0}$ is the connected component of the identity in $G$.

Proof. Consider $\left.\psi_{M}\right|_{\mathscr{A}_{\overline{0}}} \notin \pi(H)$ since $M M^{*} \in\left\{\left(\begin{array}{cc}I_{p} & 0 \\ 0 & -I_{p}\end{array}\right)\right.$, $\left.\left(\begin{array}{cc}-I_{p} & 0 \\ 0 & I_{p}\end{array}\right)\right\}$. Let $N=\left(\begin{array}{cc}X & 0 \\ 0 & Y\end{array}\right)$ such that $N N^{*} \in\left\{\left(\begin{array}{cc}I_{p} & 0 \\ 0 & -I_{p}\end{array}\right)\right.$, $\left.\left(\begin{array}{cc}-I_{p} & 0 \\ 0 & I_{p}\end{array}\right)\right\}$; then

$$
\begin{aligned}
& \left(N M^{-1}\right)\left(N M^{-1}\right)^{*}=N M^{-1}\left(M^{-1}\right)^{*} N^{*} \\
& =\left\{\begin{array}{l}
N\left(\begin{array}{cc}
I_{p} & 0 \\
0 & -I_{p}
\end{array}\right) N^{*}: \quad M M^{*}=\left(\begin{array}{cc}
I_{p} & 0 \\
0 & -I_{p}
\end{array}\right) \\
N\left(\begin{array}{cc}
-I_{p} & 0 \\
0 & I_{p}
\end{array}\right) N^{*}: M M^{*}=\left(\begin{array}{cc}
-I_{p} & 0 \\
0 & I_{p}
\end{array}\right)
\end{array}\right. \\
& =\left\{\begin{array}{l}
\left(\begin{array}{cc}
X \widetilde{X} & 0 \\
0 & -Y \widetilde{Y}
\end{array}\right): M M^{*}=\left(\begin{array}{cc}
I_{p} & 0 \\
0 & -I_{p}
\end{array}\right) \\
\left(\begin{array}{cc}
-X \widetilde{X} & 0 \\
0 & Y \widetilde{Y}
\end{array}\right): \quad M M^{*}=\left(\begin{array}{cc}
-I_{p} & 0 \\
0 & I_{p}
\end{array}\right) .
\end{array}\right.
\end{aligned}
$$

Therefore, $\left(N M^{-1}\right)\left(N M^{-1}\right)^{*} \in\left\{I_{2 p},-I_{2 p}\right\}$ and so

$$
\left.\psi_{N M^{-1}}\right|_{\mathscr{A}_{\overline{0}}}=\left.\left.\psi_{M^{-1}}\right|_{\mathscr{A}_{\overline{0}}} \psi_{N}\right|_{\mathscr{A}_{\overline{0}}} \in \pi(H) \cong \frac{H}{\operatorname{ker} \pi}
$$

Now, $\left.\psi_{N}\right|_{\mathscr{A}_{\overline{0}}}=\left.\psi_{N M^{-1} M}\right|_{\mathscr{A}_{\overline{0}}}=\left.\left.\left.\psi_{M}\right|_{\mathscr{A}_{\overline{0}}} \psi_{M^{-1}}\right|_{\mathscr{A}_{\overline{0}}} \psi_{N}\right|_{\mathscr{A}_{\overline{0}}}=$ $\left.\psi_{M}\right|_{\mathscr{A}_{\overline{0}}} \underbrace{\psi_{\mathscr{A}_{\overline{0}}}}_{N M^{-1}} \in \psi_{M} \pi(H)$. Therefore,

$$
G_{0}=\left.\pi(H) \cup \psi_{M}\right|_{\mathscr{A}_{\overline{0}}} \pi(H)
$$

\section{Conflict of Interests}

The authors declare that there is no conflict of interests regarding the publication of this paper.

\section{References}

[1] M. L. Racine, "Primitive superalgebras with superinvolution," Journal of Algebra, vol. 206, no. 2, pp. 588-614, 1998.

[2] M. L. Racine and E. I. Zelmanov, "Simple Jordan superalgebras with semisimple even part," Journal of Algebra, vol. 270, no. 2, pp. 374-444, 2003.

[3] T. Y. Lam, The Algebraic Theory of Quadratic Forms, The Benjamin/Cummings Publishing Company, 1973.
[4] A. Jaber, "Central simple superalgebras with anti-automorphisms of order two of the first kind," Journal of Algebra, vol. 323, no. 7, pp. 1849-1859, 2010.

[5] A. Jaber, "Existence of pseudo-superinvolutions of the first kind," International Journal of Mathematics and Mathematical Sciences, vol. 2008, Article ID 386468, 13 pages, 2008.

[6] W. Scharlau, Quadratic and Hermitian Forms, Springer, Heidelberg, Germany, 1985.

[7] A. Elduque and O. Villa, "The existence of superinvolutions," Journal of Algebra, vol. 319, no. 10, pp. 4338-4359, 2008.

[8] A. Weil, "Algebras with involutions and the classical groups," Indian Mathematical Society, vol. 24, pp. 589-623, 1960-1961. 


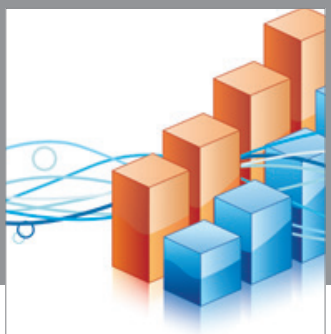

Advances in

Operations Research

vatem alat4

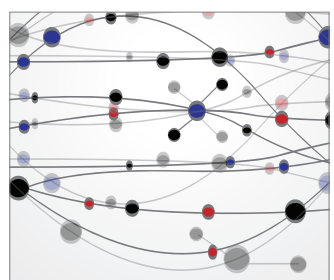

\section{The Scientific} World Journal
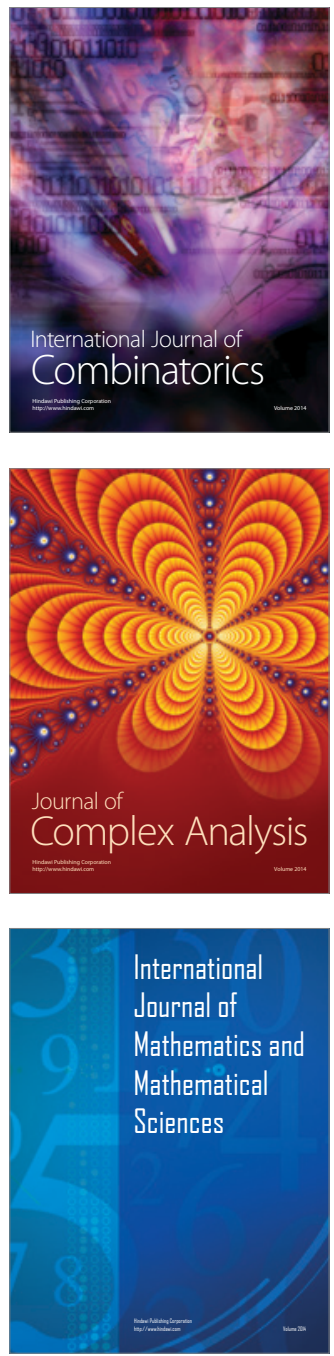
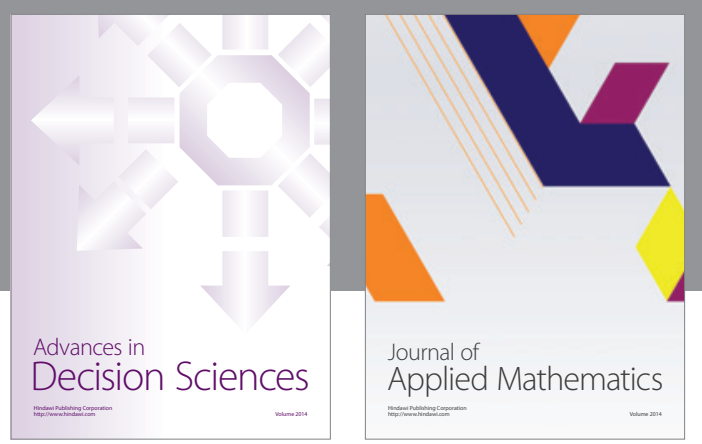

Algebra

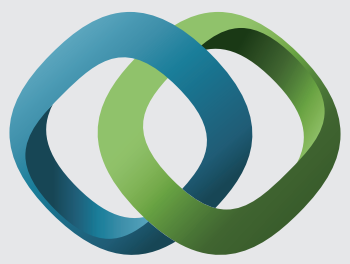

\section{Hindawi}

Submit your manuscripts at

http://www.hindawi.com
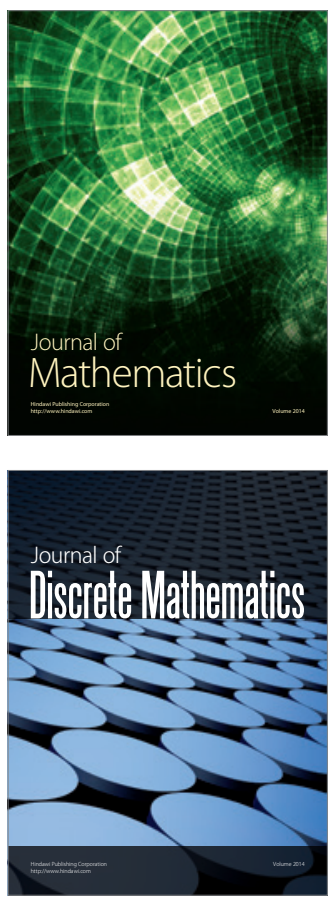

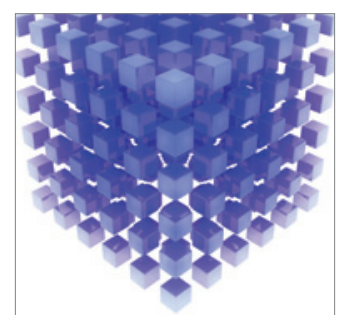

Mathematical Problems in Engineering
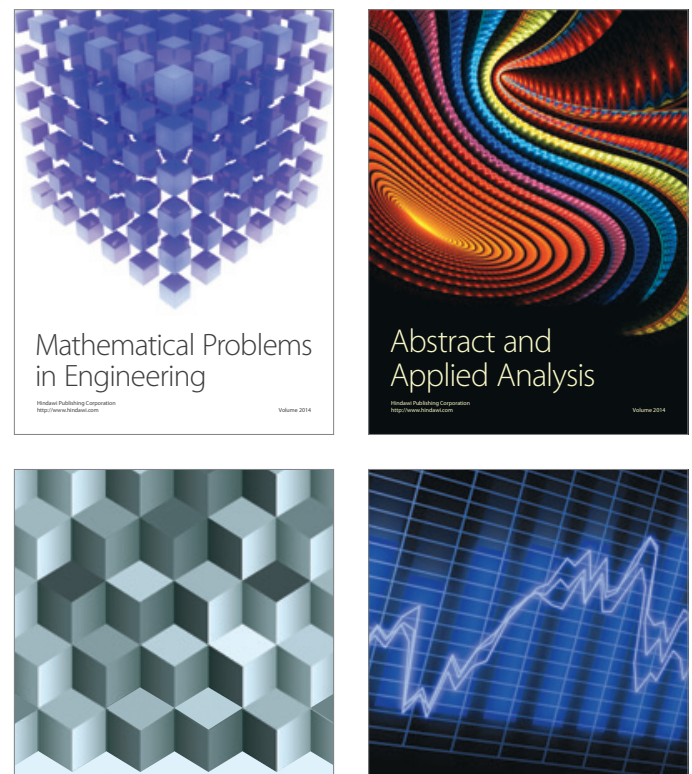

Journal of

Function Spaces

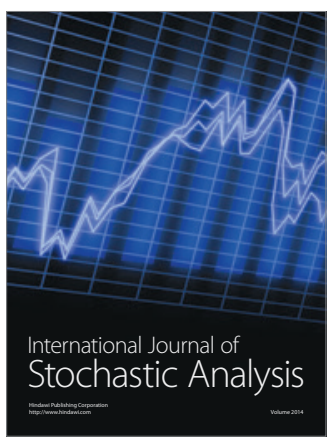

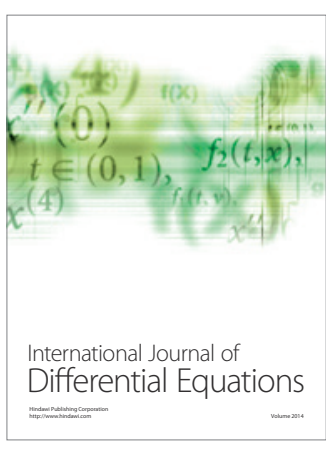
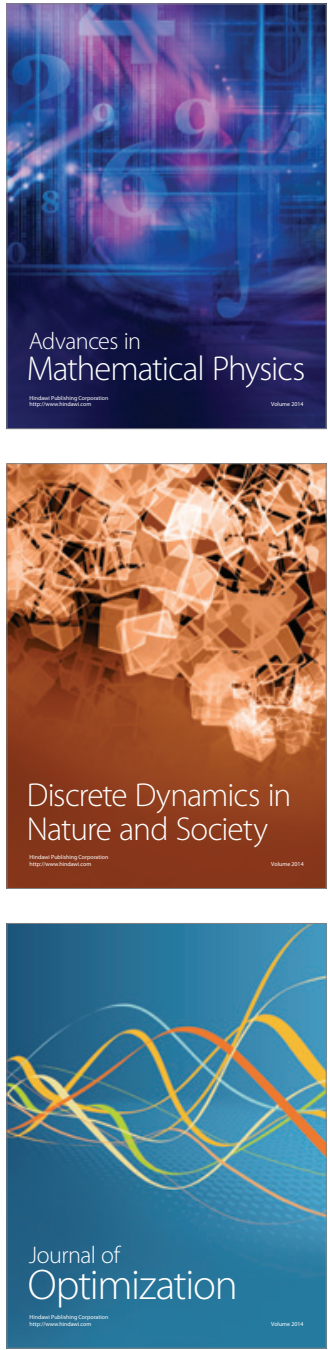